\title{
SPONTANEOUS SPINAL EPIDURAL ABSCESS FOLLOWING TRAUMA TO BACK IN A HEALTHY ADULT WITHOUT PREDISPOSING FACTORS - A RARE CASE REPORT
}

\author{
P. Shanmuga Sundaram¹, G. Ramkumar², Kalaivanan Kanniyan³ ${ }^{3}$ B. Suresh Gandhi
}

\section{HOW TO CITE THIS ARTICLE:}

P. Shanmuga Sundaram, G. Ramkumar, Kalaivanan Kanniyan, B. Suresh Gandhi. "Spontaneous Spinal Epidural Abscess Following Trauma to Back in a Healthy Adult without Predisposing Factors - A Rare Case Report". Journal of Evolution of Medical and Dental Sciences 2014; Vol. 3, Issue 08, February 24; Page: 1832-1836, DOI: $10.14260 /$ jemds/2014/2065

\begin{abstract}
Spinal epidural abscess due to its varied presentation, poses a great challenge, more so in a healthy adult, without any predisposing factors. Early diagnosis and treatment is paramount, as late diagnosis and delayed treatment result in increased mortality and morbidity. In this case report, we are presenting a case of spinal extradural abscess in a healthy young adult without predisposing factors, provisionally diagnosed as extradural hematoma and early surgical intervention confirmed the diagnosis of spinal epidural abscess and prevented any devastating consequence.
\end{abstract}

KEYWORDS: Spinal epidural abscess, extradural compression, Dorsal spine.

INTRODUCTION: The reported incidence of Spinal epidural abscess is 0.2 -2.8 cases per 10,000 hospital admissions, are associated with predisposing factors like diabetes mellitus, immunosuppressive therapy, intravenous drug abuse, malignancy ${ }^{1,2}$. Spontaneous epidural abscess in adults without predisposing factors are rare and the reported incidence is 0.88 cases per 100,000 [person years] ${ }^{3}$ and pose a diagnostic challenge to the treating physician. Definitive diagnosis and immediate treatment is required, as delayed treatment is associated with mortality of $5 \%$ and morbidity to the range of $33 \%-47 \%{ }^{4}$. Very few articles are available describing spinal epidural abscess without any predisposing factors.

Here we are reporting a case of young adult without predisposing factors presented with paraparesis following injury to the back, initially suspected of extradural hemorrhage turned out to be spinal epidural abscess.

CASE REPORT: Young adult aged 22 years presented with complaints of back pain and difficulty in walking for 7 days following injury to the back. On clinical examination, had local tenderness at D7, D8, D9, exaggerated reflexes of both lower limb and positive babinsky's sign, reduced sensation below D8. Blood investigation showed marginal increase in neutrophil count and elevated ESR. Dorsal Spine X-Ray and Magnetic resonance imaging was done as an emergency basis. Initial diagnosis of extradural hemorrhage was done as the patient had no predisposing factors and prior history of injury to the spine. Magnetic resonance imaging suggested extradural compression at D7, D8, D9 probably an extradural hemorrhage. He was advised bed rest and close monitoring of the neurological status. On second day of admission, patient was taken up for emergency surgery due to worsening of neurological symptoms. Laminectomy of D7, D8, and D9 with posterior stabilization was done. Intraoperatively greyish white soft tissue mass was noticed compressing the cord. On removal of the soft tissue, pus was noticed. Posterior pedicle instrumentation of D7, D8 and D10 and posterolateral bone grafting was done to prevent instability. Histopathological report came as an 
abscess and culture came as negative. Parental antibiotics changed to broad spectrum antibiotics and continued for 2 weeks followed with oral antibiotics for 4 weeks. Patient was made to sit and strengthening exercise of both lower limbs started, At one month follow-up patient neurological status improved to frankel Grade E. At one year follow-up patient has no recurrence of symptom.

DISCUSSION: Gradual increase in the incidence of spinal extra dual abscess has been noted over past two decades.15\%-35\% of patients with spinal epidural abscess present with prior history of injury to the back. In our case study patient had initially injury to the back and followed with native massage to the back. This trauma can produce extra dural hematoma and subsequent infection can produce extradural abscess. ${ }^{5,6}$

The classical triad of presentation is back pain, fever and neurological deficit. Only few cases presents with this classical triad. Back pain is the most common presentation followed by distal paresthesia6,7. Local tenderness of spine, with increase in erythrocyte sedimentation rate and leukocytosis, should lead to gadolinium enhanced MRI to look for spinal extradural abscess. Late diagnosis and substandard treatment are associated with high morbidity and mortality. ${ }^{4}$

David et al in his series reported a positive culture from surgical site or by intervention biopsy in $78 \%$ of cases. Often negative culture is associated with sampling after starting antibiotics. In cases of negative culture the empirical broad spectrum antibiotics can be continued. The accepted duration of treatment is 2 weeks of parenteral treatment and followed with 6 weeks of oral antibiotics. ${ }^{7}$

Spontaneous epidural abscess are usually located in the posterior to the cord. Ventrally located abscess are usually by the contiguous spread from spondylitis or discitis 5 . J.P. Grieve et al in his series had thoracic spine as the commonest site for the epidural abscess, because the dorsal spine has wide extradural space and rich venous plexus. ${ }^{7}$

Numerous articles support early surgical decompression with broad spectrum antibiotics as treatment of choice in spinal epidural abscess.5,6,8 Carefully selected patients with no neurological deficit can be treated with conservative management, provided neurological status is closely monitored and equipped with facility to do emergency decompression as sudden onset of neurological deterioration has been reported. ${ }^{8}$

The most important predictor of outcome is the neurological status of the patient at the time of surgical intervention. Patients paralyzed for more than 36 hours are less likely to improve neurologically. ${ }^{2}$

CONCLUSION: Very few percentage of patients with extradural abscess presents with classical triad of back pain, fever and neurological involvement. Even in adults without predisposing factors presenting with back pain with local spine tenderness should be viewed with high index of suspicion. Associated elevated inflammatory markers, should lead to gadolinium enhanced MRI to rule out extradural abscess. Early diagnosis and immediate management reduces the mortality and morbidity significantly. 


\section{REFERENCES:}

1. Zimmerer SME, Conen A, Müller AA, Sailer M, Taub E, Flückiger U, et al. Spinal epidural abscess: aetiology, predisponent factors and clinical outcomes in a 4-year prospective study. Eur Spine J. 2011 Dec; 20(12):2228-34.

2. Rabih O. Darouiche. Spinal Epidural Abscess. N Engl J Med. 2006 Nov; 355:2012-2020.

3. McDonald A M, Rollins JL. Spontaneous Spinal epidural abscess presenting in a previously healthy young adult man. Case Rep Med. 2013; 2013:872148.

4. Connor DE Jr, Chittiboina P, Caldito G, Nanda A. Comparison of operative and nonoperative management of spinal epidural abscess: a retrospective review of clinical and laboratory predictors of neurological outcome. J Neurosurg Spine. 2013 Jul; 19(1):119-27.

5. A R Mackenziea, R B S Lainga, C C Smitha, G F Kaarb, F W Smith. Spinal epidural abscess: the importance of early diagnosis and treatment. J Neurol Neuro surg Psychiatry 1998; 65:209-212.

6. Deardrechao, Anil nanda. Spinal Epidural Abscess: A Diagnostic Challenge. Am Fam Physician. 2002 Apr 1; 65(7):1341-1347.

7. Pradilla G, Ardila GP, Hsu W, Rigamonti D. Epidural abscesses of the CNS Lancet Neurol. 2009 Mar; 8(3):292-300.

8. Grieve J P, Ashwood N, O'Neill K S, Moore AJ. A retrospective study of surgical and conservative treatment for spinal extradural abscess. Eur Spine J. 2000 Feb; 9(1):67-71.
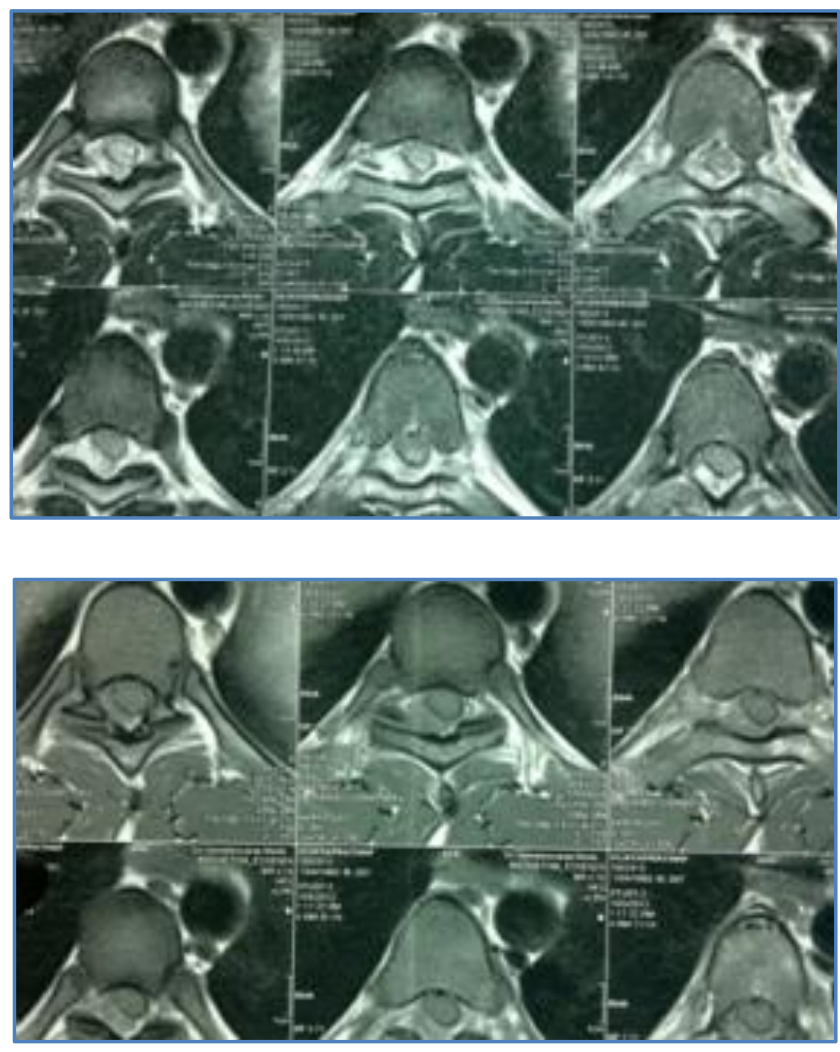

Fig. 1: Preop-hyperintense at T2 and Isointense at T1 

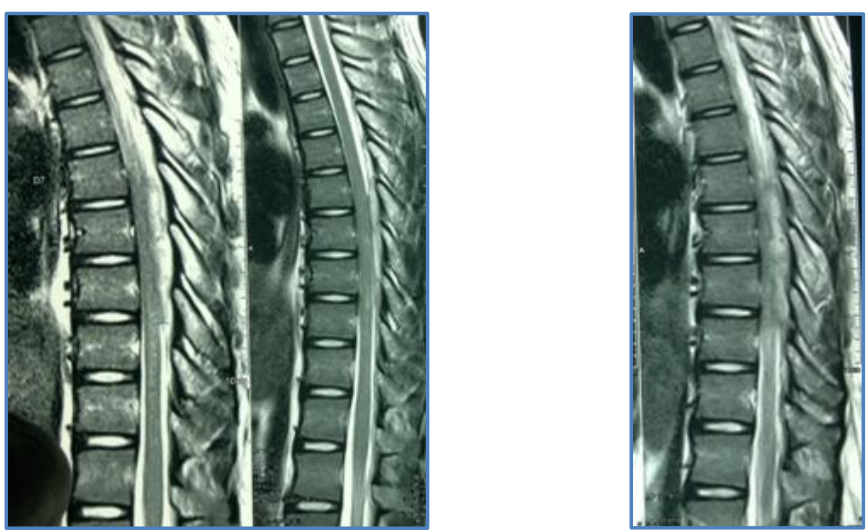

Fig. 2 : Preop images-Hyperintense at T2 and Iso intense at T1



Fig. 3 : Intraoperative picture showing pus
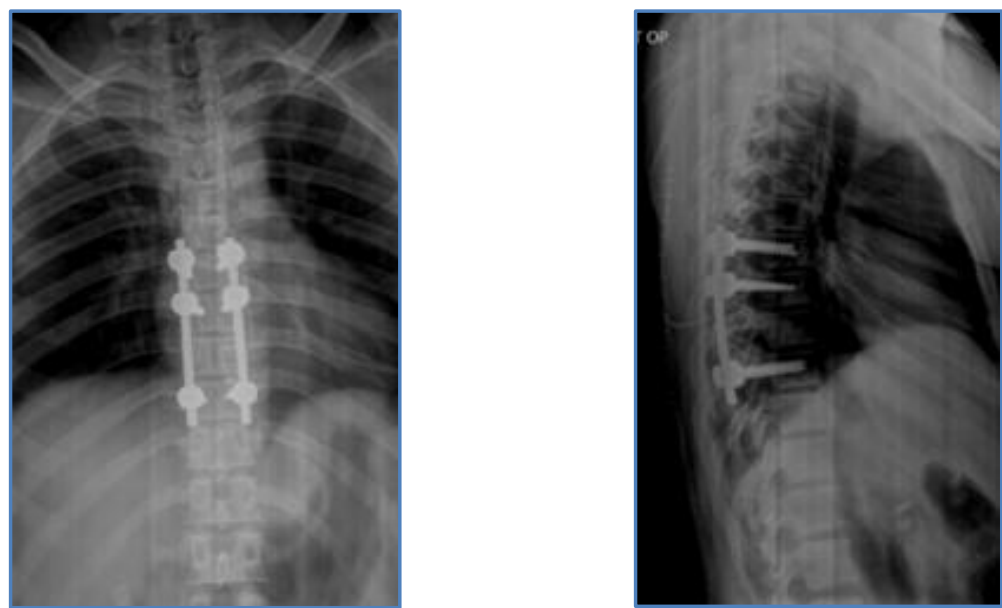

Fig. 4 : Postop X-Rays -posterior pedicle instrumentation from D7-D10 


\section{AUTHORS:}

1. P. Shanmuga Sundaram

2. G. Ramkumar

3. Kalaivanan Kanniyan

4. B. Suresh Gandhi

\section{PARTICULARS OF CONTRIBUTORS:}

1. Associate Professor, Department of Orthopaedics, Saveetha Medical College, Chennai.

2. Associate Professor, Department of Orthopaedics, Saveetha Medical College, Chennai.

3. Assistant Professor, Department of Orthopaedics, Saveetha Medical College, Chennai.
4. Assistant Professor, Department of Orthopaedics, Saveetha Medical College, Chennai.

\section{NAME ADDRESS EMAIL ID OF THE} CORRESPONDING AUTHOR:

Dr. P. Shanmuga Sundaram, 2/42, Valayapathy Salai, Mugappair East, Chennai- 600037.

E-mail: shanmu_ps@yahoo.com

Date of Submission: 05/02/2014.

Date of Peer Review: 06/02/2014.

Date of Acceptance: 12/02/2014.

Date of Publishing: 18/02/2014. 